\section{Concrete Simply Explained}

ThIs is the title of a booklet which has been published by the Society of Engineers (war-time address, 56 Church Street, Weybridge, Surrey) as reprinted from the Transactions of the Society. It was written primarily for the training of concrete operatives by Victor S. Wigmore, and presents the subject in a simple manner which should make it valuable to all grades of workers on the staff of contractors or engineers. The reader is asked to imagine that he is on a site where concreting is about to take place and $h e$ is then introduced in turn to each of the ingredients-cement, sand, coarse aggregate and water-and finally to different processes of concreting.

Each section has been treated very thoroughly and in considerable detail as, for example, in the section dealing with cement, where the reader is informed regarding the best method of storing the bags to keep them dry and to facilitate their progress to the mixers. The usual procedure of taking a sample for test, the tests applied, and the processes of setting, hardening and ripening are explained and some warnings given as to possible mistakes such as that of allowing aluminous cement and Portland cement to be mixed together. In a similarly thorough and practical manner the other ingredients and the work of concreting are described and explained. As, in the rebuilding of our towns after the War, concrete will play a very important part and there will be a demand for men who know how to make concrete of first-class quality, it is to be hoped that civil engineer. ing contractors will distribute this booklet widely among their staffs and responsible workmen.

\section{Variations of Geomagnetism}

H. D. Harradon, Department of Terrestrial Magnetism, Carnegie Institution of Washington, has given a short account of this subject ( $S k y$ and Telescope, 1, 8; June, 1942), commencing with the discovery of the secular variation by Henry Gellibrand in 1634-a discovery of considerable importance, especially for mariners. Since no observations are available prior to this date, attempts have been made to extend our knowledge of the earth's magnetism to earlier times by the study of the magnetization of eruptive rocks, baked clays and ancient potteries. In quite recent years magnetic data have been obtained from successive lava flows of Mount Etna, the assumption being that the lava retained the direction of the earth's magnetic field at the time of its solidification, but unfortunately the results have proved rather indefinite. More promising lines of research are found in the study of layers of Pleistocene clays from an old glacial lake near New Haven, Conn., and also of sediment taken from the floor of the Atlantic. In the latter case, where several thousand years of deposition were represented, there is the suggestion of a possible variation in direction of magnetization amounting to more than $60^{\circ}$-the greatest range in secular variation ever recorded. No theory up to the present has explained the mystery of these secular variations; they are probably connected with changes in the interior of the earth, regarding which there is mere speculation. The transient variations, among which the solar and lunar diurnal and the annual variations are the chief, are discussed, and a number of useful diagrams and charts illustrate the connexion between magnetic storms, auroral displays, sunspots, etc.

\section{Horticultural Work on the Amaryllidaceae}

Vor. 8 of Herbertia, the year-book for 1941 of the American Amaryllis Society (Pp. 180. Hamilton P. Traub (editor), Orlando, Florida), includes several short papers of scientific interest. W. M. James and F. T. Addicott have studied the causes of sterility in interspecific crosses within the genus Nerine, and find a possible explanation in the presence of extra chromosomes in some parents. There appears to be no correlation between chromosome numbers and flowering period, though there is a sharply marked geographical difference between early flowering species and later blooming kinds. J. Marion Shull shows that early flowering species of Hemerocallis have made their flower initials by the previous autumn, whereas later flowering kinds have no floral initiation at that time. "Food Manufacture and Flowering in the Daffodil", by Dr. John Grainger, relates climate, photosynthesis and flower formation. It is found that floral initiation begins when carbohydrate content of the bulb is maximal. The daffodil appears to provide a very efficient photosynthetic mechanism, and its carbohydrate is completely mobile. Optimum conditions for photosynthesis are provided by a high daily average of sunshine and a relatively low average temperature between $40^{\circ}$ and $50^{\circ} \mathrm{F}$. Albert W. Close directs attention to the use of sphagnum moss for the germination of seeds of difficult species, and the method has the advantage of almost eliminating the damping-off disease. Numerous shorter papers give information on various Amaryllid clones, their combination in breeding, and practical culture. The Society's score-card method for the horticultural evaluation of daylilies has been extended to Florida by John V. Watkins, Hamilton P. Traub and Wyndham Hayward

\section{Periodic Comet Forbes, 1929 ii}

Harvard College Observatory Card 626 announces that van Biesbroeck found this comet as a fifteenth magnitude object on a pair of plates exposed at the 24-in. reflector, Yerkes. Its position on 15 June -34763d. was, R.A., 0h. 04m. 25.11s., Dec. $-4^{\circ} 50^{\prime}$ 18.7". The comet showed a short tail 1 in length in position angle $270^{\circ}$. In the "Handbook of the British Astronomical Association", 1942, Mr. F. R. Cripps has given the elements of the orbit and also an ephemeris from which the object was easily found. Perihelion passage occurred only half a day earlier than was assumed.

\section{Announcements}

SIR HAROLD ScotT, director of the Bureau of Hygiene and Tropical Medicine, is retiring on August 31. The Secretary of State for the Colonies has appointed Dr. C. Wilcocks, assistant director of the Bureau, to be acting director.

THe medical faculty of the University of Basle has conferred an honorary doctorate on Prof. Eugen Pittard, rector of the University of Geneva, on the occasion of his seventy-fifth birthday, in recognition of his services to anthropology.

DR. S. F. DoREy, chief engineer surveyor of Lloyd's Register of Shipping, will deliver the Parsons Memorial Lecture of the Institute of Marine Engineers on September 16. The lecture will be on "Reduction Gearing for Marine Steam Turbines", and will be delivered at the house of the Institution of Mechanical Engineers. 